\title{
Remarks on Gross' technique for obtaining a conformal Skorohod embedding of planar Brownian motion
}

\author{
Maher Boudabra* $\quad$ Greg Markowsky ${ }^{\dagger}$
}

\begin{abstract}
In [7] it was proved that, given a distribution $\mu$ with zero mean and finite second moment, there exists a simply connected domain $\Omega$ such that if $Z_{t}$ is a standard planar Brownian motion, then $\mathcal{R} e\left(Z_{\tau_{\Omega}}\right)$ has the distribution $\mu$, where $\tau_{\Omega}$ denotes the exit time of $Z_{t}$ from $\Omega$. In this note, we extend this method to prove that if $\mu$ has a finite $p$-th moment then the first exit time $\tau_{\Omega}$ from $\Omega$ has a finite moment of order $\frac{p}{2}$. We also prove a uniqueness principle for this construction, and use it to give several examples.
\end{abstract}

Keywords: Planar Brownian motion; conformal invariance.

AMS MSC 2010: 60J65; 30C20.

Submitted to ECP on November 27, 2019, final version accepted on February 24, 2020.

\section{Introduction and statement of results}

In what follows, $Z_{t}$ is a standard planar Brownian motion starting at 0 , and for any plane domain $\Omega$ containing 0 we let $\tau_{\Omega}$ denote the first exit time of $Z_{t}$ from $\Omega$. In the elegant recent paper [7] the following theorem was proved.

Theorem 1. Given a probability distribution $\mu$ on $\mathbb{R}$ with zero mean and finite nonzero second moment, we can find a simply connected domain $\Omega$ such that $\operatorname{Re}\left(Z_{\tau_{\Omega}}\right)$ has the distribution $\mu$. Furthermore we have $E\left[\tau_{\Omega}\right]<\infty$.

We will prove several new results related to Gross' construction. Our first result is the following generalization.

Theorem 2. Given a probability distribution $\mu$ on $\mathbb{R}$ with zero mean and finite nonzero $p$-th moment (with $1<p<\infty$ ), we can find a simply connected domain $\Omega$ such that $\mathcal{R} e\left(Z_{\tau_{\Omega}}\right)$ has the distribution $\mu$. Furthermore we have $E\left[\left(\tau_{\Omega}\right)^{p / 2}\right]<\infty$.

The proof of this result depends on a number of known properties of the Hilbert transform and of the exit time $\tau_{\Omega}$, and is rather short. However the results needed are scattered through a number of different subfields of probability and analysis, and in an attempt to make the paper self-contained we have included a certain amount of exposition on these topics. We will prove the theorem in the next section.

There are several reasons why we feel that our extension is worth noting. The moments of $\tau_{\Omega}$ have special importance in two dimensions, as they carry a great deal of analytic and geometric information about the domain $\Omega$. The first major work in this

${ }^{*}$ Monash University, Australia. E-mail: maher . boudabra@monash .edu

${ }^{\dagger}$ Monash University, Australia. E-mail: gmarkowsky@gmail .com 
direction seems to have been by Burkholder in [2], where it was proved among other things that finiteness of the $p$-th Hardy norm of $\Omega$ is equivalent to finiteness of the $\frac{p}{2}$-th moment of $\tau_{\Omega}$. To be precise, for any simply connected domain $\Omega$ let

$$
\mathrm{H}(\Omega)=\sup \left\{p>0: \mathbf{E}\left[\left(\tau_{\Omega}\right)^{p}\right]<\infty\right\} ;
$$

note that $H(\Omega)$ is proved in [2, p. 183] to be exactly equal to half of the Hardy number of $\Omega$, as defined in [8], which is

$$
\tilde{\mathrm{H}}(\Omega)=\sup \left\{q>0: \lim _{r \nearrow 1} \int_{0}^{2 \pi}\left|f\left(r e^{i \theta}\right)\right|^{q} d \theta<\infty\right\},
$$

where $f$ is a conformal map from the unit disk onto $\Omega$. This equivalence was used in [2, p. 183] to show for instance that $H\left(W_{\alpha}\right)=\frac{\pi}{2 \alpha}$, where $W_{\alpha}=\{0<\operatorname{Arg}(z)<\alpha\}$ is an infinite angular wedge with angle $\alpha$. In fact, coupled with the purely analytic result [8, Thm 4.1] this can be used to determine $\mathrm{H}(\Omega)$ for any starlike domain $\Omega$. If we assume that $V$ is starlike with respect to 0 , then we may define

$$
\mathcal{A}_{r, \Omega}=\max \{m(E): E \text { is a subarc of } \Omega \cap\{|z|=r\}\},
$$

and this quantity is non-increasing in $r$ (here $m$ denotes angular Lebesgue measure on the circle). We may therefore let $\mathcal{A}_{\Omega}=\lim _{r} \nearrow_{\infty} \mathcal{A}_{r, \Omega}$, and then combining the results in [8] and [2] (see also [12]) we have $\mathrm{H}(\Omega)=\frac{\pi}{2 \mathcal{A}_{\Omega}}$. In this sense, the quantity $\mathrm{H}(\Omega)$ provides us with some sort of measure of the aperture of the domain at $\infty$. Also in [12], a version of the Phragmén-Lindelöf principle was proved that makes use of the quantity $\mathrm{H}(\Omega)$. Furthermore, the quantity $\mathbf{E}\left[\left(\tau_{\Omega}\right)^{p}\right]$ provides us with an estimate for the tail probability $P\left(\tau_{\Omega}>\delta\right)$ : by Markov's inequality, $P\left(\tau_{\Omega}>\delta\right) \leq \frac{\mathbf{E}\left[\left(\tau_{\Omega}\right)^{p}\right]}{\delta^{p}}$.

For these reasons, we would argue that Theorem 2 gives a partial answer to the following intriguing question posed by Gross in [7]: given a probability distribution $\mu$ and a corresponding $\Omega$ such that $\operatorname{Re}\left(Z_{\tau_{\Omega}}\right)$ has distribution $\mu$, in what sense are properties of $\mu$ reflected in the geometric properties of $\Omega$ ? We will have more comments on this question in the final section.

Our next result is influenced by Gross' observation that the domain corresponding to a given measure $\mu$ is not unique. Without further conditions this is correct, however we have found that natural conditions can be imposed on the domain so that a uniqueness principle holds. Before stating the result, let us make some definitions. A domain $U$ is symmetric if $\bar{z} \in U$ whenever $z \in U$. We will call a domain $U \Delta$-convex if, whenever $z_{1}, z_{2} \in U$ with $\operatorname{Re}\left(z_{1}\right)=\mathcal{R} e\left(z_{2}\right)$ then the vertical line segment connecting $z_{1}$ and $z_{2}$ lies entirely within $U$. It is straightforward to verify that any $\Delta$-convex domain is automatically simply connected. Furthermore any domain constructed by Gross' technique is both symmetric and $\Delta$-convex (see Section 2 ), and we have the following result.

Theorem 3. For any distribution $\mu$ satisfying the conditions of Theorem 2 , there is a unique domain $\Omega$ such that $\operatorname{Re}\left(Z_{\tau_{\Omega}}\right) \sim \mu$ and which is symmetric, $\Delta$-convex, and satisfies $E\left[\left(\tau_{\Omega}\right)^{p / 2}\right]<\infty$.

The importance of this result for our purposes is that it allows us to give certain solutions to the inverse problem of the one solved by Gross. That is, we can give a number of examples of domains generated by Gross' method. To be precise, if $\Omega$ is a domain which is symmetric, $\Delta$-convex, and satisfies $E\left[\left(\tau_{\Omega}\right)^{p / 2}\right]<\infty$, then it must be the domain generated by Gross' method corresponding to the distribution of $\operatorname{Re}\left(Z_{\tau_{\Omega}}\right)$. We will exploit this fact in Section 4. 


\section{Preliminaries and proof of Theorem 2}

The proof of Theorem 2 is mainly based on the Hilbert transform theory for periodic functions, and we give here a brief summary of this. For further details about the topic, we refer the reader to [3].

The Hilbert transform of a $2 \pi$ - periodic function $f$ is defined by

$$
\mathcal{H}_{f}(x):=P V\left\{\frac{1}{2 \pi} \int_{-\pi}^{\pi} f(x-t) \cot \left(\frac{t}{2}\right) d t\right\}=\lim _{\eta \rightarrow 0} \frac{1}{2 \pi} \int_{\eta \leq|t| \leq \pi} f(x-t) \cot \left(\frac{t}{2}\right) d t,
$$

where $P V$ denotes the Cauchy principal value, which is required here as the trigonometric function $t \longmapsto \cot (\cdot)$ has a simple pole at $k \pi$ with $k \in \mathbb{Z}$. Note that the more standard Hilbert transform is defined for functions $f$ on the real line by

$$
P V\left\{\frac{1}{2 \pi} \int_{-\infty}^{+\infty} \frac{f(x-t)}{t} d t\right\}
$$

However, replacing $\frac{1}{t}$ by $\cot \left(\frac{t}{2}\right)$ in the integrand is natural because $\cot \left(\frac{t}{2}\right)$ is the function which results by "wrapping" $\frac{1}{t}$ around the circle; to be precise, $\cot (\cdot)$ satisfies the following identity ([14, p. 321]):

$$
\cot (z)=\frac{1}{z}+2 z \sum_{n=1}^{+\infty} \frac{1}{z^{2}-n^{2}}=\frac{\pi}{z}+\pi \sum_{n=1}^{+\infty}\left(\frac{1}{z+\pi n}+\frac{1}{z-\pi n}\right) .
$$

In this sense, $\cot \left(\frac{t}{2}\right)$ can be seen as the periodic version of the function $\frac{1}{t}$. Let us now sketch the ideas for Gross' proof, so that we may see where the Hilbert transform comes in. We assume for now that $\mu$ has finite second moment. Let $F$ be the c.d.f of $\mu$ and consider the pseudo-inverse function of $F$ defined by

$$
G(u):=\inf \{x \in \mathbb{R} \mid F(x) \geq u\} .
$$

Note that $G$ is defined for $u \in[0,1]$. It is well known that $G\left(\operatorname{Uni}_{(0,1)}\right)$ has $\mu$ as distribution. Now consider the $2 \pi$-periodic function $\varphi$ whose restriction to $(-\pi, \pi)$ is $G\left(\frac{|\theta|}{\pi}\right)$. The map $\varphi$ is even, increasing on $(0, \pi)$ and belongs to $L^{2}$, where $L^{p}$ here and elsewhere in the paper denotes $L^{p}([-\pi, \pi])$. Thus its Fourier series is well defined and converges to $\varphi$ in $L^{2}$. We obtain hence

$$
\varphi(\theta)=\sum_{n=1}^{+\infty} \widehat{\varphi}(n) \cos (n \theta),
$$

where the $n$-th Fourier coefficient $\widehat{\varphi}(n)$ is defined for all non negative integers $n$ by $\widehat{\varphi}(n)=\frac{1}{2 \pi} \int_{0}^{2 \pi} f(t) \cos (n t) d t$. It is clear that this is the real part of the power series generated by the Fourier coefficients $\widetilde{\varphi}(z):=\sum_{n=1}^{+\infty} \widehat{\varphi}(n) z^{n}$ evaluated at $z=e^{i \theta}$; that is

$$
\mathcal{R} e\left(\widetilde{\varphi}\left(e^{i \theta}\right)\right)=\varphi(\theta) .
$$

Note that $\operatorname{Im}\left(\widetilde{\varphi}\left(e^{i \theta}\right)\right)$ is given by $\varphi(\theta)=\sum_{n=1}^{+\infty} \widehat{\varphi}(n) \sin (n \theta)$, and this is the Hilbert transform of $\varphi$. A crucial property of $\widetilde{\varphi}$, as is shown in [7], is that it is univalent. The image domain $\Omega:=\widetilde{\varphi}(\mathbb{D})$ is therefore simply connected, and it is also symmetric over the $x$-axis as $\widetilde{\varphi}(\bar{z})=\widetilde{\varphi}(z)$. Using the conformal invariance of $Z_{t}$, and the fact that $Z_{\tau_{\mathrm{D}}}$ is uniformly distributed on the boundary of $\partial \mathrm{D}$, we conclude by (2.1) that $\operatorname{Re}\left(\widetilde{\varphi}\left(Z_{\tau_{\mathrm{D}}}\right)\right)$ has distribution $\mu$. Furthermore, Parseval's identity and martingale theory implies that $E\left[\tau_{\Omega}\right]=\frac{1}{2} \sum_{n=1}^{\infty}|\widehat{\varphi}(n)|^{2}$ (see [1]), and this sum is finite since $\varphi \in L^{2}$.

Let us now see how we can extend this argument to prove Theorem 2. We will assume now that $\mu$ has a finite $p$-th moment, where $p>1$. It follows as above that $\varphi \in L^{p}$. The 
Fourier series $\sum_{n=1}^{+\infty} \widehat{\varphi}(n) \cos (n \theta)$ is still well defined and converges to $\varphi$ in $L^{p}$ ([6, Thm. 3.5.7]). Parseval's identity is no longer available to us, but the following result allows us to conclude that the Hilbert transform $\sum_{n=1}^{+\infty} \widehat{\varphi}(n) \sin (n \theta)$ of $\varphi$ is also in $L^{p}$ :

Theorem 4. [3] If $f$ is in $L^{p}$ then its periodic Hilbert transform $\mathcal{H}_{f}$ does exist almost everywhere and we have

$$
\left\|\mathcal{H}_{f}\right\|_{L^{p}} \leq \lambda_{p}\|f\|_{L^{p}}
$$

for some positive constant $\lambda_{p}$.

We remark that there are good estimates for the constant $\lambda_{p}$; see [10, Sec. 4.20, Vol. 1]. From this result we see that, as its real and imaginary parts are in $L^{p}$, the analytic function $\tilde{\varphi}(z)=\sum_{n=1}^{+\infty} \widehat{\varphi}(n) z^{n}$ lies in the Hardy space $H^{p}$, which is the space of all holomorphic maps on the disk with finite Hardy $p$-norm, defined as

$$
\|f\|_{H^{q}}:=\left\{\lim _{r \succ 1} \frac{1}{2 \pi} \int_{0}^{2 \pi}\left|f\left(r e^{i t}\right)\right|^{q} d t\right\}^{\frac{1}{q}} .
$$

$\tilde{\varphi}(z)$ is also injective, by the same argument as was used in [7, Prop. 2.2], and therefore $\Omega=\tilde{\varphi}(\mathbb{D})$ is simply connected. By Burkholder's result [2, p. 198] we have that if $f$ is a conformal function on the unit disk then the following equivalence holds:

$$
\tau_{f(\mathbb{D})} \in L^{\frac{p}{2}} \Longleftrightarrow\|f\|_{H^{p}}<\infty .
$$

We see therefore that $E\left[\left(\tau_{\Omega}\right)^{p / 2}\right]<\infty$, and the theorem is proved.

\section{Proof of Theorem 3}

In this section we prove Theorem 3, that the domain $\Omega$ generated by Gross' technique is the unique symmetric, $\Delta$-convex domain with $E\left[\left(\tau_{\Omega}\right)^{p / 2}\right]<\infty$ such that such that $\mathcal{R} e\left(Z_{\tau_{\Omega}}\right)$ has the distribution $\mu$. Before going through the proof, we need the following lemma related to the Riemann mapping theorem.

Lemma 3.1. If $U \subsetneq \mathbb{C}$ is a symmetric simply connected domain containing 0 then there exists a conformal map from $\mathbb{D}$ to $U$ such that $f(0)=0$ and $f((-1,1)) \subseteq \mathbb{R}$.

Proof. The existence of a conformal map, say $f$, from the unit disc to $U$ and sending zero to itself is guaranteed by the Riemann mapping theorem. It remains to add the constraint that $f((-1,1)) \subseteq \mathbb{R}$. Consideration of the power series shows that the map $\bar{f}(\bar{z})$ is analytic, and as $\mathbb{D}$ and $U$ are symmetric it is a conformal map from $\mathbb{D}$ to $U$. Therefore it is related to $f$ via a rotation acting on the unit disc, that is

$$
\bar{f}(\bar{z})=f\left(e^{i \theta} z\right),
$$

for some $\theta \in[0,2 \pi)$. The map $\widetilde{f}: z \longmapsto f\left(e^{i \frac{\theta}{2}} z\right)$ satisfies the requirement of the lemma since

$$
\begin{aligned}
\overline{\widetilde{f}}(\bar{z}) & =\bar{f}\left(e^{i \frac{\theta}{2}} \bar{z}\right) \\
& =\bar{f}\left(\overline{e^{-i \frac{\theta}{2}} z}\right) \\
& =f\left(e^{i \theta} e^{-i \frac{\theta}{2}} z\right) \\
& =f\left(e^{i \frac{\theta}{2}} z\right) \\
& =\widetilde{f}(z) .
\end{aligned}
$$

In particular, if $z$ is real then $\widetilde{f}(z)$ is as well, which ends the proof. 
We proceed now to prove Theorem 3. Suppose $U$ and $V$ are two domains satisfying the conditions of the theorem. Let $f: \mathbb{D} \longrightarrow U$ and $g: \mathbb{D} \longrightarrow V$ be two conformal maps fixing 0 and sending reals to reals. As $f$ and $g$ are injective, they are monotone on the real line, and we may assume then that they are increasing (if not, consider $f(-z)$ and/or $g(-z)$ instead). The power series $f(z)=\sum_{n=1}^{+\infty} a_{n} z^{n}$ and $g(z)=\sum_{n=1}^{+\infty} b_{n} z^{n}$ have real coefficients since $a_{n}=\frac{f^{(n)}(0)}{n !} \in \mathbb{R}$ and $b_{n}=\frac{g^{(n)}(0)}{n !} \in \mathbb{R}$. The fact that $E\left[\left(\tau_{U}\right)^{p / 2}\right], E\left[\left(\tau_{V}\right)^{p / 2}\right]<\infty$ implies that $\|f\|_{H^{p}},\|g\|_{H^{p}}<\infty$ (again, see [2]), and therefore the functions $f$ and $g$ have radial limits defined a.e. on $\{|z|=1\}$. That is, $f\left(e^{i \theta}\right):=\lim _{r} \gamma_{1} f\left(r e^{i \theta}\right)$ exists for Lebesque almost every $\theta$ on $[-\pi, \pi]$ (see [15, Thm 17.12] or [3]). We will compare the radial limits of $f$ and $g$ and show that they coincide a.e., but first we need another lemma.

Lemma 3.2. $Z_{\tau_{U}}$ and $Z_{\tau_{V}}$ agree in distribution with $f(X)$ and $g(X)$ respectively, where $X$ is a r.v. uniformly distributed on $\{|z|=1\}$.

Proof. Note that in applying $f$ and $g$ to $X$, we are making use of the radial limits defined above. We will prove the statement for $f$. Let $r_{n}$ be any sequence in $(0,1)$ which increases to 1 as $n \rightarrow \infty$, and let $\tau_{n}=\inf \left\{t>0:\left|Z_{t}\right|=r_{n}\right\}$. By standard martingale theory (see for instance [16, Ch. 14]), since $f\left(Z_{\tau_{n}}\right)$ is a martingale bounded in $L^{p}$ we are guaranteed the existence of a limiting r.v. $M_{\infty}$ such that $E\left[\left|f\left(Z_{\tau_{n}}\right)-M_{\infty}\right|^{p}\right] \rightarrow 0$. Therefore $f\left(Z_{\tau_{n}}\right)$ converges to $M_{\infty}$ in distribution. On the other hand, $f\left(Z_{\tau_{n}}\right)$ is equal in distribution to $f\left(X_{n}\right)$, where $X_{n}$ is any r.v. uniformly distributed on $\left\{|z|=r_{n}\right\}$. Let us choose $X_{n}$ and $X$ as follows. Let the probability space in question be the interval $[0,2 \pi)$, with probability measure given by Lebesgue measure divided by $2 \pi$. For $\omega$ in the probability space, let $X_{n}(\omega)=r_{n} e^{i \omega}$, and similarly $X(\omega)=e^{i \omega}$. By [15, Thm 17.12], we have

$$
\lim _{n \rightarrow \infty} \int_{0}^{2 \pi}\left|f\left(r_{n} e^{i \theta}\right)-f\left(e^{i \theta}\right)\right| d \theta \rightarrow 0 .
$$

Thus, $E\left[\left|f\left(X_{n}\right)-f(X)\right|\right] \rightarrow 0$, which implies that $f\left(X_{n}\right)$ converges to $f(X)$ in distribution. However, $f\left(X_{n}\right)$ and $f\left(Z_{\tau_{n}}\right)$ have the same distribution, and therefore $M_{\infty}$ and $f(X)$ agree in distribution. Now, $f\left(Z_{t}\right)$ is a time-changed Brownian motion, and therefore $f\left(Z_{\tau_{n}}\right)=\hat{Z}_{\sigma\left(\tau_{n}\right)}$, where $\sigma$ denotes the time-change and $\hat{Z}$ is a Brownian motion. By monotone convergence, $\sigma\left(\tau_{n}\right) \nearrow \tau_{U}$, and thus $f\left(Z_{\tau_{n}}\right)$ converges a.s. to $\hat{Z}_{\tau_{U}}$. It follows that $\hat{Z}_{\tau_{U}}$ is equal in distribution to $f(X)$.

$\Delta$-convexity and symmetry imply that $R e\left(f\left(e^{i \theta}\right)\right)$ and $R e\left(g\left(e^{i \theta}\right)\right)$ are a.e. even functions on $[-\pi, \pi]$ and non-increasing on $[0, \pi]$. Since $P\left(U \in\left\{e^{i \theta}:-\theta_{0}<\theta<\theta_{0}\right\}\right)=\frac{\theta_{0}}{\pi}$ for $\theta_{0} \in(0, \pi]$, it follows that for a.e. $\theta$ we must have $\operatorname{Re}\left(f\left(e^{i \theta}\right)\right)=r$, where $r$ is such that $\mu[r,+\infty)=\frac{\theta}{\pi}$, and the same must hold for $\operatorname{Re}\left(g\left(e^{i \theta}\right)\right)$. We see that $\operatorname{Re}(f)$ and $\operatorname{Re}(g)$ agree a.e. on $\{|z|=1\}$, and since $\operatorname{Im}(f), \operatorname{Im}(g)$ are obtained from these by the periodic Hilbert transform (see Section 2) we see that $f$ and $g$ agree a.e. on $\{|z|=1\} . f(z)$ and $g(z)$ for $z \in \mathbb{D}$ can be obtained from their boundary values via the Poisson integral formula ([15, Cor. 17.12]), and thus $f$ and $g$ agree. Theorem 3 is proved.

None of the three conditions in the theorem can be omitted. For example, suppose that $U=\mathbb{C} \backslash\{|\operatorname{Re}(z)| \leq 1,|\operatorname{Im}(z)| \geq 1\}$. $U$ is symmetric and $\Delta$-convex, but $E\left[\left(\tau_{\Omega}\right)^{p / 2}\right]=\infty$ for $p \geq 1$. Since $\operatorname{Re}\left(Z_{\tau_{\Omega}}\right)$ is a measure of bounded support, it will generate by Gross' method a domain $\Omega$ such that $E\left[\left(\tau_{\Omega}\right)^{p / 2}\right]<\infty$ for all $p$, and this can therefore not be equal to $U$. An example which is symmetric and has finite $p$-th moment for all $p$ but which lacks $\Delta$-convexity is displayed in Figure 2 of [7], and it is pointed out there that uniqueness fails. Finally, as will be shown below in the examples, the parabola and horizontal strip both lead to the same distribution $\mu$. Both domains are $\Delta$-convex and satisfy $E\left[\left(\tau_{\Omega}\right)^{p / 2}\right]<\infty$ for all $p>0$, but the parabola is not symmetric. This shows that the condition of symmetry cannot be omitted. On the other hand, it is interesting to note 
that both of these domains are convex, and that therefore convexity does not seem to be the correct condition for uniqueness.

\section{Examples}

In this section, we consider a series of domains and the corresponding distributions of $\operatorname{Re}\left(Z_{\tau}\right)$. In all cases that we consider the boundary of the domain will be well behaved and we will be able to find a p.d.f. of the distribution of $Z_{\tau}$ on the boundary. By this we mean that we can find a function, $\rho_{a}^{Z_{\tau}}(z)$, defined for $z$ on $\partial U$ such that for any interval $I$ on the boundary of $U$, we have $P_{a}\left(Z_{t} \in I\right)=\int_{b}^{c} \rho_{a}^{Z_{\tau}}(z(s)) d s$, where $z(s)$ is a parameterization of $\partial U$ with $\left|z^{\prime}(s)\right|=1$ and $z((b, c))=I$. We will use analytic functions and the conformal invariance of Brownian motion as our primary tool; finding exit distributions in this manner has previously been considered in [13], and following the convention there we will use the notation $\rho_{a}^{Z_{\tau}}(z) d s$ to denote this density, with the $d s$ to indicate that the curve $z(s)$ is parameterized by arclength.

If we have found the p.d.f of $Z_{\tau}$ on $\partial U$, then we can deduce the p.d.f's of $X_{\tau}$ and $Y_{\tau}$ provided that the boundary of the domain is smooth enough in the sense that, locally around $z=x+y i$, we have

$$
y=\varphi_{z}(x),
$$

for some differentiable bijective function $\varphi_{z}$. To see how, let $x$ be an element of $\{\mathcal{R} e(z) \mid z \in \partial D\}$. Since a positive infinitesimal element $d z \in \partial D$ is expressed as $d z=\sqrt{d x^{2}+d y^{2}}$, then

$$
\begin{aligned}
\rho_{\mathcal{R} e(a)}^{X_{\tau}}(x) d x & =\sum_{\mathcal{R} e(z)=x} \rho_{a}^{Z_{\tau}}(z) d z \\
& =\sum_{\mathcal{R} e(z)=x} \rho_{a}^{Z_{\tau}}(x+y i) \sqrt{d x^{2}+d y^{2}} \\
& =\sum_{\mathcal{R} e(z)=x} \rho_{a}^{Z_{\tau}}\left(x+\varphi_{z}(x) i\right) \sqrt{1+\varphi_{z}^{\prime}(x)^{2}} d x .
\end{aligned}
$$

Finally we get

$$
\begin{aligned}
& \rho_{\mathcal{R} e(a)}^{X_{\tau}}(x)=\sum_{\mathcal{R} e(z)=x} \rho_{a}^{Z_{\tau}}\left(x+\varphi_{z}(x) i\right) \sqrt{1+\left\{\frac{d \varphi_{z}}{d x}(x)\right\}^{2}} \\
& \rho_{\mathcal{I} m(a)}^{Y_{\tau}}(y)=\sum_{\mathcal{I} m(z)=y} \rho_{a}^{Z_{\tau}}\left(\varphi_{z}^{-1}(y)+y i\right) \sqrt{1+\left\{\frac{d \varphi_{z}^{-1}}{d y}(y)\right\}^{2}} .
\end{aligned}
$$

Notice that that both sets $\{z \mid \mathcal{R} e(z)=x\}$ and $\{z \mid \mathcal{I} m(z)=y\}$ are countable due to (4.1), which justifies the sum symbols in (4.2). This proves the formula for the distribution of $X_{\tau}$, and $Y_{\tau}$ can of course be obtained similarly. The following diagram, which should be viewed at the infinitesimal level, provides the intuitive justification for the formulas.

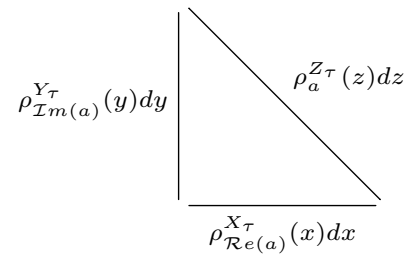

Before going through examples, we recall a lemma from [13] which we will use to find the exit distribution of Brownian motion from various domains. Let $\gamma$ be any smooth 
curve parameterized by arclength, $B_{t}$ a Brownian motion starting at $a$, and $\tau$ a stopping time such that $B_{\tau} \in \gamma$ a.s. $\rho_{\tau}^{a}(w) d s$ will denote the density of $B_{\tau}$ on $\gamma$, when it exists, with $d s$ denoting the arclength element. The lemma we require is as follows.

Lemma 4.1. [13, Th. 2] Let $U$ be a domain, and suppose $f$ is a function analytic on $U$. Let $B_{t}$ be a Brownian motion starting at $a$, and $\tau$ a stopping time such that the set of Brownian paths $\left\{B_{t}: 0 \leq t \leq \tau\right\}$ lie within $U$ a.s. Suppose that $\gamma$ is a smooth curve in $U$ such that $B_{\tau} \in \gamma$ a.s. Then for any $a \in U$ and $w \in f(\gamma)$ we have

$$
\rho_{\hat{\tau}}^{f(a)}(w) d s=\sum_{z \in f^{-1}(w) \cap \gamma} \frac{\rho_{\tau}^{a}(z)}{\left|f^{\prime}(z)\right|} d s
$$

In each case below, the stopping time $\tau$ will be the exit time of a domain; note that this does not conflict with the requirement that $\gamma \subseteq U$, since the analytic function $f$ in our examples will always be a function which is analytic on a domain strictly containing the closure of $U$, and the theorem can be applied in this larger domain. We will proceed by applying this lemma to find the exit distribution of various domains, and then projecting these onto the real line using (4.3).

\subsection{Unit disc}

If $Z_{t}$ starts at zero at stopped at $\tau_{\mathrm{D}}$ then due to the rotational invariance of the Brownian motion $Z_{\tau_{\mathrm{D}}}$ is uniformly distributed on the circle, i.e

$$
\rho_{0}^{Z_{\tau_{\mathrm{D}}}}\left(e^{\theta i}\right)=\frac{1}{2 \pi} .
$$

Using the unit circle equation $x^{2}+y^{2}=1$, we extract the distributions of $X_{\tau_{\mathrm{D}}}$ and $Y_{\tau_{\mathrm{D}}}$ on $(-1,1)$ :

$$
\begin{aligned}
\rho_{0}^{X_{\tau_{\mathrm{D}}}}(x) & \stackrel{(4.2)}{=} \sum_{z \in\left\{x \pm i \sqrt{1-x^{2}}\right\}} \rho_{a}^{Z_{\tau}}(z) \\
& =\frac{1}{2 \pi} \sqrt{1+\left(\frac{x}{1-x^{2}}\right)^{2}}+\frac{1}{2 \pi} \sqrt{1+\left(-\frac{x}{1-x^{2}}\right)^{2}} \\
& =\frac{1}{\pi \sqrt{1-x^{2}}} .
\end{aligned}
$$

Similarly for $\rho_{0}^{Y_{\tau_{\mathrm{D}}}}(y)$. We remark that $X_{\tau_{\mathrm{D}}}$ and $Y_{\tau_{\mathrm{D}}}$ follow the scaled and centered Arc-sine law on $(-1,1)$ (see [5, p. 49]). If the starting point is $a=u+v i \neq 0$, then the distribution of $Z_{\tau_{\mathrm{D}}}$ is given by

$$
\rho_{a}^{Z_{\tau_{\mathrm{D}}}}\left(e^{\theta i}\right) d \theta=\frac{1-|a|^{2}}{2 \pi\left|1-\bar{a} e^{\theta i}\right|^{2}} d \theta ;
$$

see [13, Ex. 1]. Using the coordinates expressions $(x, y)=(\cos \theta, \sin \theta)$, we find the distributions of $X_{\tau_{\mathrm{D}}}$ and $Y_{\tau_{\mathrm{D}}}$ :

$$
\rho_{u}^{X_{\tau_{\mathrm{D}}}}(x)=\frac{1-|a|^{2}}{2 \pi \sqrt{1-x^{2}}}\left(\frac{1}{\mid 1-\bar{a}\left(x+\left.\sqrt{1-x^{2}} i\right|^{2}\right.}+\frac{1}{\mid 1-\bar{a}\left(x-\left.\sqrt{1-x^{2}} i\right|^{2}\right.}\right)
$$

and

$$
\rho_{v}^{Y_{\tau}}(y)=\frac{1-|a|^{2}}{2 \pi \sqrt{1-y^{2}}}\left(\frac{1}{\mid 1-\bar{a}\left(\sqrt{1-y^{2}}+\left.y i\right|^{2}\right.}+\frac{1}{\mid 1-\bar{a}\left(-\sqrt{1-y^{2}}+\left.y i\right|^{2}\right.}\right) .
$$

In particular we recover $\rho_{\mathcal{I} m(a)}^{Y_{\tau_{\mathrm{D}}}}(y)=\rho_{\mathcal{R} e(-a i)}^{X_{\tau_{\mathrm{D}}}}$. 


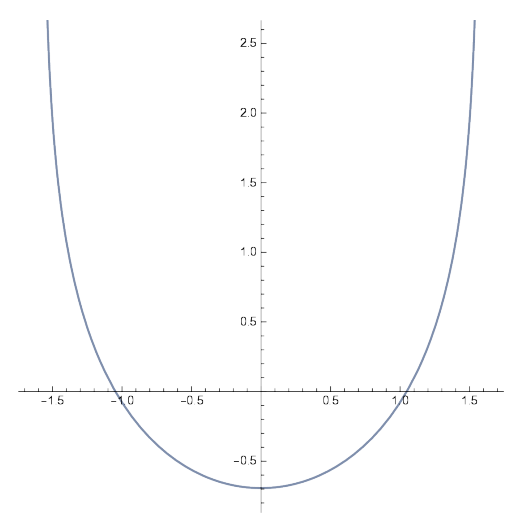

Figure 4.1: $\mathscr{P}$ has two vertical asymptotes, namely at $\pm \frac{\pi}{2}$

\subsection{Catenary}

The following example was brought to our attention by Hugo Panzo and Phanuel Mariano, and is the subject of their interesting preprint [11]. The map $f(z)=-i \ln (1+z)$ maps the unit disc to the domain $\mathscr{P}$ shown in Figure 4.1.

If we set $z=e^{\theta i}$ and $w=f(z)$ then $w=x+y i=\arctan \left(\frac{\sin \theta}{1+\cos \theta}\right)-\frac{i}{2} \ln (2+2 \cos \theta)$. It is not hard to check that $x=\theta / 2$, and thus

$$
y=-\frac{1}{2} \ln (2+2 \cos (2 x))
$$

which explains the asymptotes at $x= \pm \frac{\pi}{2}$. It is straightforward to verify that $\frac{d x}{d y}=$ $\sqrt{\frac{1}{4 e^{2 y}-1}}$, and using Lemma 4.1 and (4.2) we get

$$
\begin{aligned}
\rho_{0}^{Y_{\tau \mathscr{P}}}(y) & =\frac{2\left|e^{w i}\right|}{2 \pi} \sqrt{1+\left(\sqrt{\frac{1}{4 e^{2 y}-1}}\right)^{2}} \\
& =\frac{e^{-y}}{\pi} \sqrt{\frac{4 e^{2 y}}{4 e^{2 y}-1}} \\
& =\frac{2}{\pi} \sqrt{\frac{1}{4 e^{2 y}-1}} .
\end{aligned}
$$

Note that the factor 2 in the first equation comes from the fact that each value on the $y$-axis great than $-\ln 2$ has two preimages on the curve. Note that if we rotate $P$ a quarter turn to the left it is $\Delta$-convex and symmetric, so this is (a rotation of) the domain generated by Gross' method for this distribution. On the other hand, this is not the case for the real part, in fact

$$
\begin{aligned}
\rho_{0}^{X_{\tau_{\mathscr{P}}}(x)} & =\frac{\left|e^{w i}\right|}{2 \pi} \sqrt{1+\left(\frac{\sin 2 x}{1+\cos 2 x}\right)^{2}} \\
& =\frac{e^{-y}}{2 \pi} \sqrt{1+\left(\frac{\sin 2 x}{1+\cos 2 x}\right)^{2}} \\
& =\frac{\sqrt{2+2 \cos 2 x}}{2 \pi} \sqrt{\frac{2}{1+\cos 2 x}} \\
& =\frac{1}{\pi} .
\end{aligned}
$$




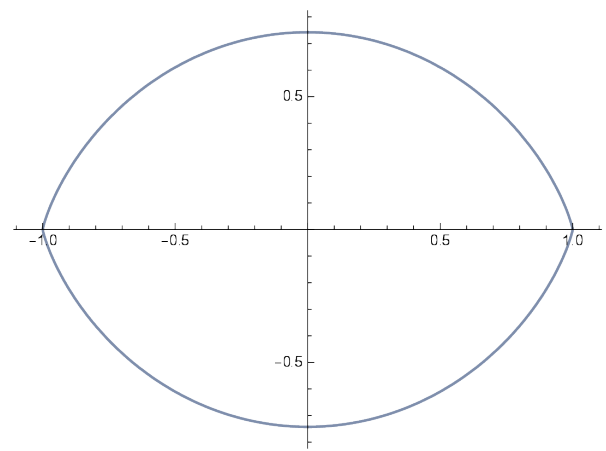

Figure 4.2: The symmetric and $\Delta$-convex domain generated by Uni $(-1,1)$.

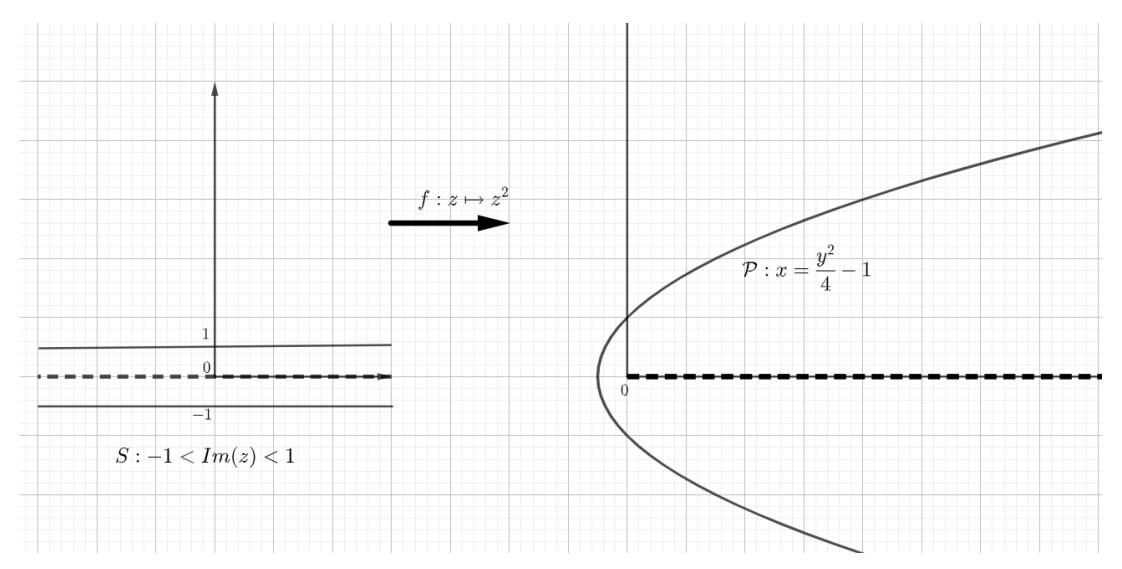

Figure 4.3: Action of $z \longmapsto z^{2}$ on the strip.

So $X_{\tau_{\mathscr{P}}}$ is uniformly distributed over $(-\pi / 2, \pi / 2)$. However, this is not the domain generated by Gross' method for the uniform distribution, as it is not symmetric over the real axis. An approximation of that domain is illustrated in Figure 4.2, which also appears in [7] and [11].

Incidentally, [11] contains a great deal more information about this example, as well as another proof that the exit distribution of this domain is uniform when projected onto the real axis.

\subsection{Parabola}

Let $S$ be the horizontal strip $\{z,-1<\operatorname{Im}(z)<1\}$ and $\mathcal{P}=f(S)$ where $f: z \longmapsto z^{2}$.The map $f$ is not conformal as it is 2 to 1 , however it maps the boundary $\partial S$ to $\partial \mathcal{P}$. That is

$$
\partial \mathcal{P}=\left\{(u, v) \mid u=x^{2}-1, v= \pm 2 x, x \in \mathbb{R}\right\},
$$

so $\mathcal{P}$ is the area limited by the parabola of the equation

$$
x=\frac{y^{2}}{4}-1 .
$$

The following image may help the reader visualize how the map works. Note that the real axis is mapped to the nonnegative real axis, and each of the strips $\{z,-1<$ $\operatorname{Im}(z)<0\},\{z, 0<\operatorname{Im}(z)<1\}$ are "bended" into the interior of the parabola minus the nonnegative real axis. 
The p.d.f of $Z_{\tau_{S}}$ starting from the origin is given by

$$
\rho_{0}^{\tau_{S}}(z=x \pm i)=\frac{\operatorname{sech}\left(\frac{\pi}{2} x\right)}{4},
$$

where $\operatorname{sech}(z)=\frac{2}{e^{z}+e^{-z}}$ is the hyperbolic secant function. The distribution of $X_{\tau_{\Omega}}$ is equally shared between the two horizontal lines of the boundary of the strip because of symmetry, and therefore admits the density $\frac{\operatorname{sech}\left(\frac{\pi}{2} x\right)}{2} d x$. (4.6) can be proved by conformal invariance ([13, Ex. 4]) or as a consequence of the optional stopping theorem ([4, Prop. 2]).

The expression of $\rho_{0}^{Z_{\tau_{\mathcal{P}}}}$ is

$$
\begin{aligned}
\rho_{0}^{Z_{\tau_{\mathcal{P}}}}(w=u+v i) & =\sum_{z \in f^{-1}\{w\}} \frac{\rho_{0}^{Z_{\tau_{S}}}(z)}{\left|f^{\prime}(z)\right|} \\
& =\frac{\rho_{0}^{Z_{\tau_{S}}\left(\frac{v}{2}+i\right)}}{\left|f^{\prime}\left(\frac{v}{2}+i\right)\right|}+\frac{\rho_{0}^{Z_{\tau_{S}}}\left(-\frac{v}{2}+i\right)}{\left|f^{\prime}\left(-\frac{v}{2}+i\right)\right|} \\
& =2 \frac{\rho_{0}^{Z_{\tau_{S}}}\left(\frac{v}{2}+i\right)}{\left|f^{\prime}\left(\frac{v}{2}+i\right)\right|} \\
& =\frac{\operatorname{sech}\left(\frac{\pi}{4} v\right)}{4 \sqrt{\frac{v^{2}}{4}+1}},
\end{aligned}
$$

where $w \in \partial \mathcal{P}$. Via (4.3), we get for $(u, v) \in(-1,+\infty) \times \mathbb{R}$

$$
\begin{aligned}
\rho_{0}^{X_{\tau_{\mathcal{P}}}}(u) & =\rho_{0}^{Z_{\tau_{\mathcal{P}}}}(u+\sqrt{4 u+4} i) \sqrt{1+\frac{4}{4 u+4}}+\rho_{0}^{Z_{\tau_{\mathcal{P}}}}(u-\sqrt{4 u+4} i) \sqrt{1+\frac{4}{4 u+4}} \\
& =\frac{\operatorname{sech}\left(\frac{\pi}{2} \sqrt{u+1}\right)}{2 \sqrt{u+1}},
\end{aligned}
$$

and

$$
\begin{aligned}
& \rho_{0}^{Y_{\tau_{\mathcal{P}}}}(v)=\rho_{0}^{Z_{\tau_{\mathcal{P}}}}\left(\frac{v^{2}}{4}-1+v i\right) \sqrt{1+\frac{v^{2}}{4}} \\
& =\frac{\operatorname{sech}\left(\frac{\pi}{4} v\right)}{4} \text {. }
\end{aligned}
$$

It is a surprising fact that this agrees with the density obtained from the strip $\{z,-2<\operatorname{Im}(z)<2\}$, as can be verified by applying Lemma 4.1 with the map $f(z)=2 z$, which takes $\{z,-1<\operatorname{Im}(z)<1\}$ to $\{z,-2<\operatorname{Im}(z)<2\}$. However, as mentioned in the previous section, this does not contradict Theorem 3 since it is the distribution of $\operatorname{Im}\left(Z_{\tau_{\mathcal{P}}}\right)$, and $\mathcal{P}$ is not symmetric or $\Delta$-convex with respect to the imaginary axis.

\subsection{Ellipse of the form $\frac{x^{2}}{\cosh ^{2} R}+\frac{y^{2}}{\sinh ^{2} R}=1$}

This example leads to a complicated distribution, but is included because it illustrates how our method can be applied to maps which are infinite to one. Let $E$ be the centered ellipse of equation $\frac{x^{2}}{\cosh ^{2} R}+\frac{y^{2}}{\sinh ^{2} R}=1$. Although not every ellipse can be expressed in this form, it does capture every possible ratio between major and minor axes, and therefore any ellipse can be expressed simply as a scaling of one of these, with the corresponding $\mu$ 's being scalings of each other as well. A conformal map from the disk onto the ellipse is known but is not simple ([9]); however, as is shown in [13, Thm. 2] the map in question does not need to be injective as long as it maps the boundary of its domain of definition onto the boundary of the target domain. It turns out that the 
holomorphic function $f(z)=\sin (z)$ maps the horizontal strip $S_{R}:=\{z, R<\operatorname{Im}(z)<-R\}$ onto $E$. This is how it works: for $z=x+R i$ we have

$$
\begin{aligned}
\sin (z) & =\frac{e^{(x+R i) i}-e^{-(x+R i) i}}{2 i} \\
& =\frac{e^{-R}(\cos x+i \sin x)-e^{R}(\cos x-i \sin x)}{2 i} \\
& =\left(\frac{e^{R}+e^{-R}}{2}\right) \sin x+i\left(\frac{e^{R}-e^{-R}}{2}\right) \cos x \\
& =\cosh R \sin x+i \sinh R \cos x .
\end{aligned}
$$

So if we set $\sin z=u+v i$ then $\frac{u^{2}}{\cosh ^{2} R}+\frac{v^{2}}{\sinh ^{2} R}=1$. Thus, $f$ maps the $\operatorname{lines}\{\operatorname{Im}(z)=$ $R\}$ and $\operatorname{Im}(z)=-R$ \} onto the curve $\frac{x^{2}}{\cosh ^{2} R}+\frac{y^{2}}{\sinh ^{2} R}=1$, and it follows that the interior of the strip is mapped onto the interior of the ellipse. Now let $w \in \partial E_{a, b}$ and $\rho_{E}(w)$ be the p.d.f of $Z_{\tau_{E}}$, then

$$
\begin{aligned}
\rho_{E}(w=u+v i) d w & =\sum_{z \in f^{-1}\{w\}} \frac{\rho Z_{\tau}(z)}{|\cos (z)|} d w \\
& =\sum_{z \in f^{-1}\{w\}} \frac{\operatorname{sech}\left(\frac{\pi x}{2 R}\right)}{2 R|\cos (z)|} d w .
\end{aligned}
$$

If we assume $v \geq 0$, then since $u=\cosh R \sin x$, we may take $x=\arcsin \left(\frac{u}{\cosh R}\right)+2 \pi n$ (the other possible values of $x$ for a given $u$ correspond to $v<0$ ). Thus,

$$
\begin{aligned}
\rho_{E}(w=u+v i) & d w=\sum_{n \in \mathbb{Z}} \frac{\operatorname{sech}\left(\frac{\pi}{2 R} \arcsin \left(\frac{u}{\cosh R}\right)+\frac{n \pi^{2}}{R}\right)}{2 R\left|\cos \left(\arcsin \left(\frac{u}{\cosh R}\right)+2 n \pi\right)\right|} d w \\
= & \frac{1}{2 R\left|\cos \left(\arcsin \left(\frac{u}{\cosh R}\right)\right)\right|} \sum_{n \in \mathbb{Z}} \operatorname{sech}\left(\frac{\pi}{2 R} \arcsin \left(\frac{u}{\cosh R}\right)+\frac{n \pi^{2}}{R}\right) d w \\
= & \frac{\cosh R}{2 R \sqrt{\cosh ^{2} R-u^{2}}} \sum_{n \in \mathbb{Z}} \operatorname{sech}\left(\frac{\pi}{2 R} \arcsin \left(\frac{u}{\cosh R}\right)+\frac{n \pi^{2}}{R}\right) d w .
\end{aligned}
$$

We can now project this density onto the real and imaginary axes as before, using $\frac{d v}{d u}=\frac{-u \sinh R}{\cosh R \sqrt{\cosh ^{2} R-u^{2}}}$, to get

$$
\begin{aligned}
& \rho_{0}^{X_{\tau_{H}}}(u)=\frac{\cosh R \sqrt{1+\frac{u^{2} \sinh ^{2} R}{\cosh ^{2} R\left(\cosh ^{2} R-u^{2}\right)}}}{2 R \sqrt{\cosh ^{2} R-u^{2}}} \sum_{n \in \mathbb{Z}} \operatorname{sech}\left(\frac{\pi}{2 R} \arcsin \left(\frac{u}{\cosh R}\right)+\frac{n \pi^{2}}{R}\right) d u, \\
& \rho_{0}^{Y_{\tau_{H}}}(v)=\frac{\sinh R \sqrt{1+\frac{v^{2} \cosh ^{2} R}{\sinh ^{2} R\left(\sinh ^{2} R-v^{2}\right)}}}{2 R \sqrt{\sinh ^{2} R-v^{2}}} \sum_{n \in \mathbb{Z}} \operatorname{sech}\left(\frac{\pi}{2 R} \arcsin \left(\frac{v}{\sinh R}\right)+\frac{n \pi^{2}}{R}\right) d v .
\end{aligned}
$$

\subsection{Right part of the Hyperbola $x^{2}-y^{2}=1$}

If $R:=\{z \mid \operatorname{Re}(z)>1\}$ then the p.d.f of $Z_{\tau_{R}}$ started at $a=\delta+\eta i \in R$ is given by [13, Ex. 2]

$$
\rho_{a}^{\tau_{R}}(1+y i)=\frac{(\delta-1)}{\pi|1+i y-a|^{2}} d y .
$$

The square function $s: z \longmapsto z^{2}$ maps the right part limited by the hyperbola $x^{2}-y^{2}=1$, say $H$, to $R$. Therefore for every $z=x+y i \in \partial H$

$$
\begin{aligned}
\rho_{\sqrt{a}}^{\tau_{H}}(z) d z & \stackrel{z^{2}=1+v i}{=}\left|s^{\prime}(\sqrt{1+v i})\right| \rho_{a}^{\tau_{R}}(1+v i) \\
& =2(\delta-1) \frac{\sqrt{x^{2}+y^{2}}}{\pi|1+v i-a|^{2}} d z \\
& =2(\delta-1) \frac{\sqrt{x^{2}+y^{2}}}{\pi|1-a+2 x y i|^{2}} d z .
\end{aligned}
$$


In particular if $a$ is real, and by using the relation $x^{2}-y^{2}=1$, we get the densities of $X_{\tau_{H}}$ and $Y_{\tau_{H}}$ :

$$
\begin{aligned}
& \rho_{\sqrt{a}}^{X_{\tau_{H}}}(x)=\frac{2(\delta-1)}{\pi} \frac{2 x^{2}-1}{\sqrt{x^{2}-1}}\left\{\frac{1}{\left|2 x \sqrt{x^{2}-1} i+1-a\right|^{2}}+\frac{1}{\left|2 x \sqrt{x^{2}-1} i-1+a\right|^{2}}\right\}, \\
& \rho_{\sqrt{a}}^{Y_{\tau_{H}}}(y)=\frac{2(\delta-1)}{\pi} \frac{2 y^{2}+1}{\sqrt{1+y^{2}}\left|2 y \sqrt{y^{2}+1} i+1-a\right|^{2}} .
\end{aligned}
$$

We note that it is known that $E\left[\tau_{H}^{p}\right]<\infty$ precisely when $p<1$; see [2, (4.2)]. Also by [2, Thm. 2.1], $E\left[\tau_{H}^{p}\right]<\infty$ precisely when $E\left[\left(Z_{\tau_{H}}\right)^{2 p}\right]<\infty$, and thus when $p<1$. This is straightforward to verify from the previous equation, as for example the formula for $\rho_{\sqrt{a}}^{Y_{\tau_{H}}}$ is asymptotic to $y^{-3}$ at $\infty$.

\section{Concluding remarks}

We do not know whether Theorem 2 holds for $\frac{1}{2} \leq p \leq 1$. There are many difficulties to proving the result in this range. One is that the analogue of Theorem 4 does not hold, even for $p=1$; for a counterexample, see [10, p. 212, Vol. 2]. Furthermore $H^{p}$ and $L^{p}$ are not as well behaved for $p<1$; their respective norms are not true norms, for instance, as the triangle inequality fails. In any event, regardless of the veracity of the theorem for $\frac{1}{2} \leq p \leq 1$, one should certainly exercise extreme caution in attempting to extend it to $p<\frac{1}{2}$. This is because for any simply connected domain $\Omega$ strictly smaller than $\mathbb{C}$ itself we have $E\left[\left(\tau_{\Omega}\right)^{p / 2}\right]<\infty$ for any $p<\frac{1}{2}$; this is proved in [2]. Thus a measure with infinite $p$-th moment for some $p<\frac{1}{2}$ cannot correspond in this manner to a simply connected domain.

The question posed by Gross in [7] on how properties of $\mu$ are reflected in the geometry of $\Omega$ is, in our opinion, an interesting one. We emphasize in this regard that we have now shown that every $\Delta$-convex, symmetric domain can be obtained uniquely from a probability distribution, so in this context we would hope that geometric conditions will translate directly to probabilistic ones. Gross proposed finding a condition which forced $\Omega$ to be convex; this appears difficult, especially considering that according to Gross' simulations the domain corresponding to a Gaussian is not convex. We would like therefore to suggest several weaker properties that $\Omega$ might have, and propose that finding sufficient conditions on $\mu$ for these might be interesting problems.

- $\Omega$ is starlike with respect to 0 .

- $\sup _{z \in \Omega}|\operatorname{Im}(z)|<\infty$. That is, $\Omega$ is contained in an infinite horizontal strip. Note that this would imply that all moments of $\mu$ are finite, because all moments of the exit time of a strip are finite, but that this is not sufficient: if $\Omega$ is the parabolic region $\left\{x>y^{2}-1\right\}$, then all moments of $\tau_{\Omega}$ are finite (proof: $\Omega$ can fit inside a rotated and translated wedge $W_{\alpha}$ with arbitrarily small aperture $\alpha$, and therefore its exit time is dominated by that of the wedge, which can have finite $p$-th moment for as large $p$ as we like) but $\sup _{z \in \Omega}|\mathcal{I} m(z)|=\infty$.

- $\lim \sup _{|\mathcal{R} e(z)| \rightarrow \infty, z \in \Omega}|\operatorname{I} m(z)|=0$.

\section{Acknowledgements}

We would like to thank Zihua Guo, Paul Jung, Wooyoung Chin, and an anonymous referee for helpful comments.

\section{References}

[1] R. Bañuelos and T. Carroll. Brownian motion and the fundamental frequency of a drum. Duke Mathematical Journal, 75(3):575-602, 1994. MR-1291697 
[2] D. Burkholder. Exit times of Brownian motion, harmonic majorization, and Hardy spaces. Advances in Mathematics, 26(2):182-205, 1977. MR-0474525

[3] P. Butzer and R. Nessel. Hilbert transforms of periodic functions. In Fourier Analysis and Approximation, pages 334-354. Springer, 1971. MR-0510857

[4] W. Chin, P. Jung, and G. Markowsky. Some remarks on invariant maps of the Cauchy distribution. Statistics and Probability Letters, 158, 2020. MR-4025681

[5] W. Feller. An introduction to probability theory and its applications. 1957, 2. MR-0088081

[6] L. Grafakos. Classical Fourier analysis, volume 2. Springer, 2008. MR-2445437

[7] R. Gross. A conformal Skorokhod embedding. Electronic Communications in Probability, 24(68):1-11, 2019. MR-4029437

[8] L. Hansen. Hardy classes and ranges of functions. The Michigan Mathematical Journal, 17(3):235-248, 1970. MR-0262512

[9] S. Kanas and T. Sugawa. On conformal representations of the interior of an ellipse. 31(2):329, 2006. MR-2248819

[10] F. King. Hilbert transforms. Cambridge University Press Cambridge, 2009. MR-2542215

[11] P. Mariano and H. Panzo. Conformal skorokhod embeddings of the uniform distribution and related extremal problems. arXiv:2001.12008, 2020.

[12] G. Markowsky. The exit time of planar Brownian motion and the Phragmén-Lindelöf principle. Journal of Mathematical Analysis and Applications, 422(1):638-645, 2015. MR-3263480

[13] G. Markowsky. On the distribution of planar Brownian motion at stopping times. Annales Academiæ Scientiarum Fennicæ Mathematica, 2018. MR-3839827

[14] R. Remmert. Theory of complex functions, volume 122. Springer Science \& Business Media, 2012. MR-1084167

[15] W. Rudin. Real and complex analysis. Tata McGraw-Hill, 2006. MR-0344043

[16] D. Williams. Probability with martingales. Cambridge university press, 1991. MR-1155402 


\section{Electronic Journal of Probability Electronic Communications in Probability}

\section{Advantages of publishing in EJP-ECP}

- Very high standards

- Free for authors, free for readers

- Quick publication (no backlog)

- Secure publication $\left(\mathrm{LOCKSS}^{1}\right)$

- Easy interface (EJMS²)

\section{Economical model of EJP-ECP}

- Non profit, sponsored by $\mathrm{IMS}^{3}, \mathrm{BS}^{4}$, ProjectEuclid ${ }^{5}$

- Purely electronic

\section{Help keep the journal free and vigorous}

- Donate to the IMS open access fund ${ }^{6}$ (click here to donate!)

- Submit your best articles to EJP-ECP

- Choose EJP-ECP over for-profit journals

\footnotetext{
${ }^{1}$ LOCKSS: Lots of Copies Keep Stuff Safe http://www. lockss.org/

${ }^{2}$ EJMS: Electronic Journal Management System http://www.vtex.lt/en/ejms.html

${ }^{3}$ IMS: Institute of Mathematical Statistics http://www.imstat.org/

${ }^{4}$ BS: Bernoulli Society http://www. bernoulli-society.org/

${ }^{5}$ Project Euclid: https://projecteuclid.org/

${ }^{6}$ IMS Open Access Fund: http://www.imstat.org/publications/open.htm
} 